\title{
Crystalline lens imaging during accommodation with a slit- scanning tomography system: preliminary results
}

\author{
Jessica Gomes and Sandra Franco \\ Centre of Physics, University of Minho, 4710-057, Braga, Portugal
}

\begin{abstract}
A slit-scanning tomography system previously developed in the Centre of Physics of University of Minho allows to capture images of the eye and posteriorly calculus of the biometric (thickness and curvature) and optical (wavefront aberrations) properties of the corneal and crystalline lens surfaces. It is intended to optimise the system for an accurate computing of these properties of the crystalline lens in the relaxed state and during ocular accommodation. In this study, preliminary experimental results of the crystalline lens imaging of a subject during accommodation are presented.
\end{abstract}

\section{Introduction}

The human eye is an optical system with different refractive components where the cornea and crystalline lens are the most important and determine the optical quality of the eye. [1]

In order to obtain a clear image of objects, at different distances, on the retina, it is necessary that the lens changes its position, shape, and thickness, called this process ocular accommodation. [2] These alterations cause changes in the optical quality of the eye. [3]

Previous studies claimed that the ocular aberrations have impact on determining the accommodative direction and influence the precision of the accommodative response. [4] However, there is no in-depth knowledge about the impact of the biometric and optical changes of the lens in the ocular accommodation.

The study of the changes in the lens during accommodation and their consequent influence on the characteristics of the accommodative response can provide important information of the accommodative process, in healthy eyes and in dysfunctional accommodative responses. Moreover, it can be helpful to evaluate the results of intraocular lenses implantation and its efficiency.

Over the last years, an optical tomography system was developed in the centre of physics of University of Minho, which allow to obtain images of the anterior ocular segment and do its $3 \mathrm{D}$ reconstruction. It is possible to calculate its thickness, curvature and the wavefront aberrations. This system was later also applied to the lens in relaxed state. [5-9]

The purpose of this work was to optimize the system to obtain accurate information of the crystalline lens, both in the relaxed state and during ocular accommodation. It is intended to make the system able to study the changes in the lens changes during accommodation.

\section{Material and Methods}

\subsection{Slit-scanning tomography system}

This system (figure 1.A) contains two systems; a lighting system, which projects a beam of light in the anterior segment of the eye, scanning and allowing different optical sections to be obtained; and an optical system, which obtains the images of the optical sections.

An algorithm developed for this purpose (figure 1.B) allows analysing these images and calculating the biometric parameters, such as the thickness and curvature of the cornea and lens, and the optical properties, i.e., the wave front aberrations.
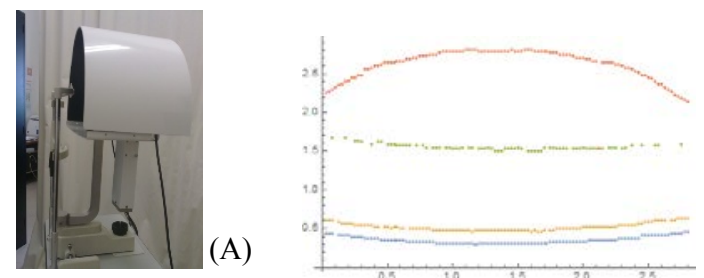

Fig. 1. (A) Slit-scanning tomography system. (B) Example of a mathematical adjustment for the calculation of biometric and optical properties of the anterior (blue) and posterior (orange) corneal surfaces and anterior (green) and posterior (red) crystalline lens surfaces.

\subsection{Data collection procedure}

The study adhered to the tenets of the Declaration of Helsinki and was approved by the Ethical Subcommission of Life and Health Science of University of Minho. After the explanation of the procedures, an informed consent was obtained from the subject.

In this preliminary study, measurements were taken on a subject without mydriatic medication.

* Corresponding author: sfranco@fisica.uminho.pt 
The subject was asked to fix a distant point with the left eye, maintaining his accommodation relaxed, while the images were captured in the right eye. After measurements in the relaxed state, the accommodation was stimulated in $4.00 \mathrm{D}$, placing a negative spherical lens in front of the left eye, while images continued to be captured in the contralateral eye.

The images of the eye's subject with and without accommodation were analysed in the previously developed algorithm and the curvature radius and power of the anterior surface of the crystalline lens in both conditions were calculated.

\section{Results}

\subsection{Imaging with the slit-scanning tomography system}

The images of the subject's eye captured by the slitscanning tomography system in the relaxed state and with $4.00 \mathrm{D}$ of accommodative stimulus are shown in figure 2 . It was possible to identify the anterior and posterior surfaces of the cornea and the anterior surface of the lens.
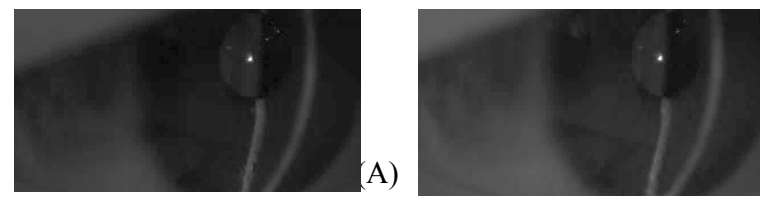

Fig. 2. Image captured with the tomography system of the subject's eye unaccommodated (A) and accommodated (B).

(B)

\subsection{Image processing of the crystalline lens}
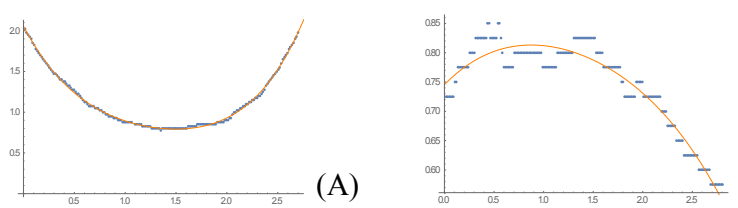

Fig. 3. Edge detection and curve fitting of the anterior surface of the lens unaccommodated (A) and accommodated (B).

\subsection{Changes in properties of the crystalline lens with} accommodation

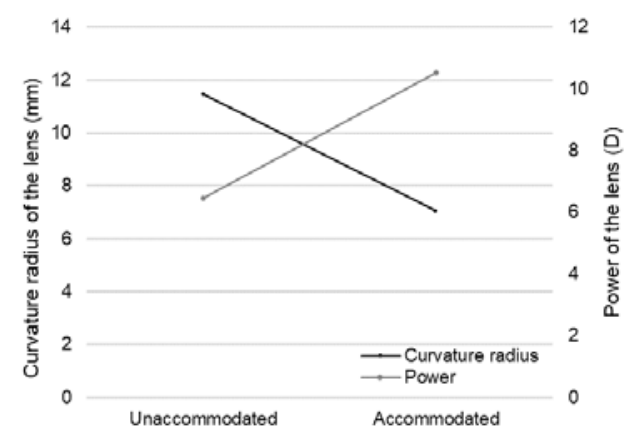

Fig. 4. Curvature radius and power of the lens in relaxed state and with an accommodative stimulus of $4.00 \mathrm{D}$.

The central curvature radius and power of the anterior surface of the lens were obtained for the unaccommodated and accommodated state (Fig 4). For an accommodative stimulus of $4.00 \mathrm{D}$, the subject accommodated $4.05 \mathrm{D}$, reducing the curvature radius of the lens from $11.46 \mathrm{~mm}$ to $7.04 \mathrm{~mm}$.

\section{Conclusions}

The slit-scanning tomography system allows the detection of the changes with accommodation of the biometric parameters of the crystalline lens, such as curvature radius and power. As it was excepted, in this preliminary study the anterior surface of the lens presented smaller curvature radius and greater power when the accommodation was being stimulated.

It is intended to use this system to perform a 3D reconstruction of the lens and obtain the changes with accommodation of other properties, such as the thickness and wavefront aberrations.

\section{Acknowledges}

This work was supported by the Portuguese Foundation for Science and Technology (FCT) in the framework of the Strategic Funding UID/FIS/04650/2019 and by the project PTDC/FIS-OTI/31486/2017 and POCI-01-0145-FEDER031486.

\section{References}

1. P. Artal, A. Benito, J. Tabarnero. The human eye is an example of robust optical design. J Vis $\mathbf{6}, 1$ (2006)

2. Y. Wang, Y. Shao, Y. Yuan. Simultaneously measuring ocular aberrations and anterior segment biometry during accommodation. J Innov Opt Heal Sci 8, 1550005 (2015)

3. J. He, S. Burns, S. Marcos. Monochromatic aberrations in the accommodated human eye. Vision Res 40, 41-48 (2000)

4. E. Fernández, P. Artal. Study of the effects of monochromatic aberrations in the accommodation response by using adaptive optics. J Opt Soc Am A 22, 1732-1738 (2005)

5. S. Franco, J. Almeida, M. Parafita. Optical corneal tomography. In mastering the techniques of IOL power calculations, ed. A Garg, J E Hoyos, D Dementiev, 21-23. New Delhi: Jaypee Brothers Medical Publishers (P) Ltd (2009)

6. S. Franco, J. Almeida, M. Parafita. Corneal thickness and elevation maps computed from optical rotary scans. J Refract Surg 20, S576-S580 (2004)

7. S. Franco, J. Almeida, M. Parafita. Measuring corneal thickness with a rotary scanning system. J Refract Surg 18, S630-S633 (2002)

8. C. Oliveira, J. Almeida, S. Franco. Crystalline lens imaging with a slit-scanning system. In Technology and Medical Sciences 93-96 (2011)

9. C. Oliveira, J. Almeida, S. Franco. Imaging of aphakic intraocular lens with a slit-scanning tomography system. In International Conference on AOP - International Society for Optics and Photonics 8001, 800135 (2011) 